\section{Biosynthesis of Triacylglycerols (TAGs) in plants and algae}

\author{
Alexandro Cagliari, ${ }^{1}$ Rogerio Margis, ${ }^{2,3}$ \\ Felipe dos Santos Maraschin, ${ }^{4}$ \\ Andreia Carina Turchetto-Zolet, ${ }^{1,3}$ \\ Guilherme Loss, ${ }^{3}$ \\ Marcia Margis-Pinheiro, \\ 'Departamento de Genética, \\ 2Departamento de Biofísica, \\ ${ }^{3}$ Centro de Biotecnologia, ${ }^{4}$ Departamento \\ de Botânica, Universidade Federal do Rio \\ Grande do Sul, Brazil
}

\section{Abstract}

Triacylglycerols (TAGs), which consist of three fatty acids bound to a glycerol backbone, are major storage lipids that accumulate in developing seeds, flower petals, pollen grains, and fruits of innumerous plant species. These storage lipids are of great nutritional and nutraceutical value and, thus, are a common source of edible oils for human consumption and industrial purposes. Two metabolic pathways for the production of TAGs have been clarified: an acyl CoA-dependent pathway and an acyl-CoA-independent pathway. Lipid metabolism, specially the pathways to fatty acids and TAG biosynthesis, is relatively well understood in plants, but poorly known in algae. It is generally accepted that the basic pathways of fatty acid and TAG biosynthesis in algae are analogous to those of higher plants. However, unlike higher plants where individual classes of lipids may be synthesized and localized in a specific cell, tissue or organ, the complete pathway, from carbon dioxide fixation to TAG synthesis and sequestration, takes place within a single algal cell. Another distinguishing feature of some algae is the large amounts of very long-chain polyunsaturated fatty acids (VLC-PUFAs) as major fatty acid components. Nowadays, the focus of attention in biotechnology is the isolation of novel fatty acid metabolizing genes, especially elongases and desaturases that are responsible for PUFAs synthesis, from different species of algae, and its transfer to plants. The aim is to boost the seed oil content and to generate desirable fatty acids in oilseed crops through genetic engineering approaches. This paper presents the current knowledge of the neutral storage lipids in plants and algae from fatty acid biosynthesis to TAG accumulation.

\section{Introduction}

The present review describes the current understanding of the fatty acid and triacylglycerol (TAG) biosynthesis in vascular plants and algae. The two initial sections compare fatty acid profiles and the pathways for TAG accumulation present in these organisms, emphasizing the peculiarities of each group. The third section summarizes some economical applications of TAG molecules. In addition, it discusses some biotechnological efforts aimed at the genetic manipulation of fatty acids and TAG content for the production of nutritionally and industrially desirable oils in crop plants and algae. Other classes of lipids, such as carotenoids that also belong to the lipid class of compounds, are not discussed here. For other lipid classes, readers can report to recent reviews on algae ${ }^{1-3}$ and on vascular plants..$^{4-8}$

\section{Fatty acid biosynthesis in plants}

Triacylglycerols (TAGs), as a highly reduced form of carbon, are an important energy reserve in plant seeds, providing nutrients for subsequent germination and seedling development. These storage lipids are of great nutritional and nutraceutical value, and a common source of edible oils for human consumption and industrial purposes. ${ }^{9}$

TAGs of most seeds usually contain the same acyl groups that are found in membrane lipids: palmitic acid (16:0), stearic acid (18:0), oleic acid $\left(18: 1 \Delta^{9}\right)$, linoleic acid $\left(18: 2 \Delta^{9,12}\right)$, and $\alpha$-linolenic $\left(18: 3 \Delta^{9,12,15}\right) .{ }^{10}$ These fatty acids are often referred to as common fatty acids. ${ }^{11}$ The biosynthesis of these five major fatty acids occurs primarily in two subcellular compartments: the de novo synthesis (de novo synthesis is defined as the synthesis of complex molecules from simple molecules, as opposed to being recycled after partial degradation; in such cases, synthesis of fatty acids from acetyl-CoA, a non-fatty acid precursor) of palmitic and stearic acids and the desaturation of stearic acid to oleic acid occur in plastids, while the conversion of oleic acid to linoleic and then $\alpha$-linolenic occurs in endoplasmic reticulum (ER). ${ }^{12,13}$

In plastids, fatty acids are synthesized from acetyl-Coenzyme A (acetyl-CoA) in a threestep process: i) irreversible carboxylation of acetyl-CoA by the action of acetyl-CoA carboxylase to form malonyl-CoA. Subsequently, the malonyl group is transferred to acyl carrier protein (ACP) giving rise to malonyl ACP, the primary substrate of the fatty acid synthase complex (Figure 1A). ${ }^{14}$ The formation of malonyl $\mathrm{CoA}$, catalyzed by the highly regulated plastidic acetyl CoA carboxylase complex, is the committed step in fatty acid synthesis. ${ }^{15}$; ii) repeated condensation of malonyl-CoA with a growing
Correspondence: Márcia Pinheiro Margis, Departamento de Genética, Universidade Federal do Rio Grande do Sul, Prédio 43312, 91501-970, Porto Alegre, Brasil.

Tel. +55.51.3308.9814

E-mail: marcia.margis@ufrgs.br

Key words: fatty acid biosynthesis, TAG accumulation, lipid metabolism.

Acknowledgments: this project was supported by CNPq (Conselho Nacional de Desenvolvimento Científico e Tecnológico), CAPES (Coordenação de Aperfeiçoamento de Pessoal de Nível Superior), FAPERGS (Fundação de Amparo a Pesquisa do Estado do Rio Grande do Sul), FINEP (Financiadora de Projetos) and MCT (Ministério de Ciência e Tecnologia).

Contributions: AC is principal author in the conception, design, analysis and interpretation of data, and drafted the article. RM participated in the conception and design, and critically revised the article. FM, AT-Z and GL revised the article and contributed important intellectual content. MM-P participated in conception and design and final approval of the version to be published.

Received for publication: 28 March 2011.

Revision received: 14 July 2011.

Accepted for publication: 11 October 2011

This work is licensed under a Creative Commons Attribution NonCommercial 3.0 License (CC BYNC 3.0).

(C)Copyright A. Cagliari et al., 2011

Licensee PAGEPress, Italy

International Journal of Plant Biology 2011; 2:e10 doi:10.4081/pb.2011.e10

ACP-bound acyl chain by action of the fatty acid synthase complex, with the consecutive addition of two carbon units for each elongation cycle to form 16:0-ACP (Figure 1A). ${ }^{14}$ For each cycle, four separated reactions are necessary. The first step corresponds to the formation of 3-ketobutyl-ACP through the condensation of acetyl-CoA with malonyl-ACP by ketoacyl synthase III (KAS III), followed by reduction to 3-hydroxylacyl-ACP, dehydration to an enoylACP and a second reduction to form the elongated 4:0-ACP. Subsequent rounds of condensation reactions of 4:0-ACP with malonyl-ACP giving rise 14:0-ACP and 16:0-ACP are catalyzed by KAS I enzyme. ${ }^{16}$; the elongation of 16:0-ACP to form 18:0-ACP, catalyzed by KAS II and the first desaturation step (that occurs in the plastid) where a $\Delta^{9}$-desaturase is the enzyme responsible for the conversion of 18:0ACP to 18:1-ACP (Figure 1A). These three fatty acids (16:0-ACP, 18:0-ACP and 18:1-ACP) are then exported to the cytosol into the acyl-CoA and acyl-lipid pools. ${ }^{9,17}$ In some organisms, the 
elongation process can be extended and fatty acid chains containing up to 18 carbon atoms can be synthesized. ${ }^{14}$

The termination of fatty acid elongation is catalyzed by acyl ACP thioesterases (acyl-ACP hydrolases) enzymes. Thioesterases catalyzes the hydrolysis of acyl-ACP to produce free fatty acids, which are able to cross the plastidial envelope to be reactivated as acyl-CoAs on the outside of the organelle (Figure 1A). ${ }^{18}$ In plants, two main thioesterase types were described: the FatA class, which preferentially removes oleate from $\mathrm{ACP}$, and FatB thioesterases that are active with saturated and unsaturated acyl ACPs, and, in some species, with shorter-chain-length acyl ACPs. ${ }^{19-21}$ The interplay between the fatty acid synthase complex, $\Delta^{9}$ desaturase, and the two thioesterases determines the ratio of acyl chains produced in the plastid for TAG formation. ${ }^{10}$

After it has been exported from the plastids, oleic acid enters the cytosolic pool (Figure 1A) and is imported into the ER in association with CoA. Oleic acid is then available for conversion to linoleic and $\alpha$-linolenic acid by the sequential action of substrate-specific desaturases. . $14,17,22,23$ Alternatively, other fatty acid chain modifications can occur in ER. For example, in Castor bean (Ricinus communis L.), oleic acid undergoes a hydroxylation process, through the activity of the oleate hydroxylase (FAH12) enzyme, yielding ricinoleic acid (C18:0H), an unusual hydroxylated $(\mathrm{OH})$ fatty acid. ${ }^{22}$ Finally, part of the fatty acids produced in plastids and modified in the ER is retained as structural components of cellular membranes (phospholipids of the ER and galactolipids of plastids, for instance) and the rest is transferred to TAG and accumulate as an energy source. ${ }^{17}$

\section{Unusual fatty acids}

Differently from the conservative fatty acid composition observed in the plant membrane lipids, many evolutionarily divergent angiosperm species accumulate substantial amounts of unusual acyl chains in their seedstorage lipids. About 300 naturally occurring different fatty acids have been described in seed oils. However, it has been estimated that thousands more could be present throughout the plant kingdom. ${ }^{10,11}$

Unusual fatty acids correspond to those presenting chemical structures that deviate from the common fatty acids found in the majority of plant species. The structures of the unusual fatty acid can vary in chain length from 8 to 24 carbons. Alternatively, they can present modifications along acyl chain composition, like double bonds in unusual positions or novel functional groups (such as hydroxy, epoxy, cyclic, halogen or an acetylenic group). ${ }^{11}$
A)

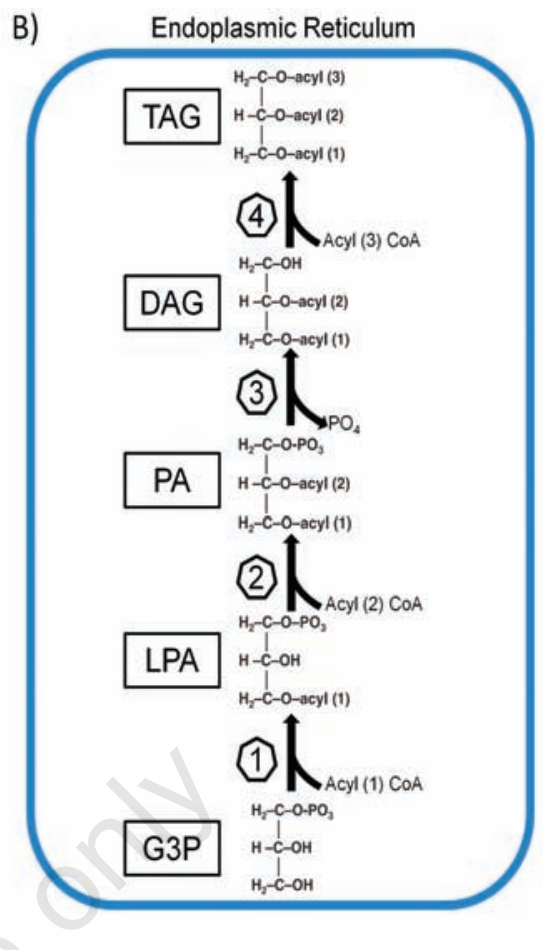

Figure 1. Fatty acid biosynthesis and Kennedy pathway for triacylglycerol biosynthesis in plants. (A) Fatty acid synthesis occurs in plastids through repeated steps of condensation by the action of several enzymes that compound the fatty acid synthase complex (FAS), which promotes the consecutive addition of two carbon units for each elongation cycle to form 16:0. Then, the elongation of 16:0 occurs to form 18:0 and the first desaturation step where 18:0 originates 18:1. The termination of fatty acid elongation is catalyzed by thioesterases enzymes and these three fatty acids are then exported to the cytosol into the acyl-CoA pools and are able to be accumulated as TAG molecules. (B) Kennedy pathway starts with acylation of glycerol-3-phosphate (G3P) to form lysophosphatidic acid (LPA) through the action of $s n$-glycerol-3-phosphate acyltransferase (1). The second acyl-CoA dependent acylation is catalyzed through the catalytic action of lysophosphatidic acid acyltransferase (2), leading to the formation of phosphatidic acid (PA). Phosphatidic acid phosphatase (3) catalyzes the release of phosphate from PA to produce DAG. The final acylation is driven by diacylglycerol acyltransferase (4) that converts DAG into TAG.

In several plant species, unusual fatty acids are the predominant fatty acids represented in the seed oil composition. The reason for such diversity observed in seed oil constituents is unknown, but plants seem to be able to tolerate high levels of unusual fatty acids in storage lipids because they are rapidly sequestered into oil bodies and, therefore, have no structural roles. The physical and chemical properties of many unusual fatty acids might explain, at least in part, why they are excluded from the membrane lipids of seeds, and are absent from other parts of the plant. It is hypothesized that they would disturb the structural integrity of the membrane bilayer and have deleterious effects on the cell. Consequently, storage and membrane lipids have different fatty acid compositions. $^{11}$

Nature contains a wide variety of unusual fatty acids, some of which are important for industry and human health. Producing these unusual fatty acids in agronomical suitable plants has been a long standing goal for com- panies and researchers involved in the field of oilseed engineering. ${ }^{24-27}$

\section{Saturated acyl chain fatty acids}

Species of Araceae, Lauraceae, Lythraceae and Ulmaceae often contain saturated acyl chain lengths ranging from $\mathrm{C} 8$ to $\mathrm{C} 14$. Coconut oil, for example, possesses more than $90 \%$ of its acyl chains composed by saturated fatty acids, generally with fatty acids chain lengths ranging from $\mathrm{C} 8$ to $\mathrm{C} 16$, predominantly lauric acid (12:0). Another example includes Brassicaceae, which shows a preferential production of very long chain saturated fatty acids with carbon lengths of $\mathrm{C} 20-\mathrm{C} 24 .{ }^{10}$

\section{Medium-chain fatty acids}

Some species of oleaginous plants are able to accumulate high amounts of medium-chain fatty acids, generally showing fatty acid chains with less than 16 carbons (Figure 2A). ${ }^{17}$

For common fatty acid formation, the grow- 
ing acyl chain is terminated when it reaches a length of 16 or 18 carbons, by the action of chain-length-specific thioesterases, ${ }^{28-30}$ which cleaves the acyl group from the ACP to produce a free fatty acid, thus terminating their elongation. Plants that synthesize medium-chain fatty acids have an additional acyl-ACP thioesterase enzyme, which is responsible for the premature cleavage of the acyl-chain from ACP, redirecting fatty acid synthesis from long (C16-C18) to medium (C8-C14) chains. ${ }^{11}$

Examples of the most commercially important medium-chain fatty acid oils include the palm kernel and coconut oils, which contain predominantly lauric acid (12:0) (Figure 2A) in their fatty acid compositions. ${ }^{17}$ In addition, several species of the genus Cuphea are also known to accumulate high amounts of medium-chain fatty acids, ranging from C8 to C14, usually with only one chain length dominating in each species. ${ }^{31}$

\section{Very long chain poly unsaturated fatty acids}

Plant membrane sphingolipids contain significant amounts of very long chain FAs (VLCFA) that possess fatty acid chain lengths ranging from 20 to 26 carbons (Figure 2B). Unsaturated VLC-FAs are also found in the storage oils of some plants (Cruciferae species) and in epicuticular and storage wax esters. They are synthesized outside of plastids by successive rounds of elongation of a $\mathrm{C} 18$ fatty acyl precursor by two carbons originating from malonyl-Co $\mathrm{A}^{11}$ by a membrane-bound elongase complex. ${ }^{32}$ In comparison, very long chain polyunsaturated fatty acids (VLCPUFAs), defined as fatty acids containing 20 or more carbon atoms and three or more double bonds, are almost completely absent in higher plants. $^{33,34}$ The biosynthesis of VLC-PUFAs consists of cycles of alternated desaturation and chain elongation, in which the desaturation reactions occur on acyl-PC substrates and the elongation reactions occur on acyl-CoA. ${ }^{17}$

The primary biosynthesizing sources of VLC-PUFAs are marine microorganisms such as algae, which represent the base of an aquatic food network that results in the accumulation of VLC-PUFAs in fish oils. ${ }^{35,36}$ However, some fungi and lower plants can synthesize VLC-PUFAs, and animals can convert dietary fatty acids such as linoleic and $\alpha$-linolenic acids to these more complex forms. ${ }^{26,34,37,38}$ From a nutritional point of view, the most important VLC-PUFAs are arachidonic (ARA; $\omega 6-20: 4 \Delta^{5,8,11,14}$ ), eicosapentaenoic (EPA; $\omega 3$ $\left.20: 5 \Delta^{5,8,11,14,17}\right)$, and docosahexaenoic acid (DHA; $\omega 322: 6 \Delta^{4,7,10,13,16,19}$ ). VLC-PUFAs are not only required as components of membrane phospholipids in specific tissues or as precursors for the synthesis of the different groups of
A)

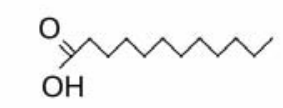

Medium-chain-fatty acid Lauric (12:0)

B)

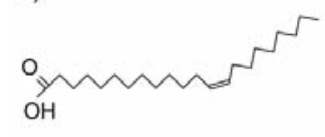

Double-bound position

Petroselinic (18:1 $\Delta 6)$

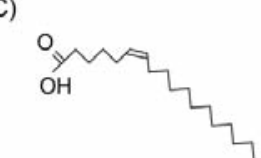

D)

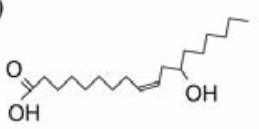

Hydroxy-fatty acid Ricinoleic (18:1-OH)

Very-long-chain-fatty acid
Erucic $(22: 1)$

E)

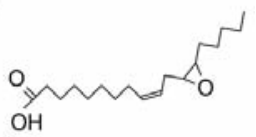

Epoxy-fatty acid Vernolic (18:1 $=0)$

Figure 2. Examples of unusual fatty acids produced by plants. (A) Medium-chain-fatty acid. (B) Very-long-chain-fatty acid. (C) Unusual double-bound position fatty acid. (D) Hydroxy-fatty acid. (E) Epoxy-fatty acid.

eicosanoid effectors, but also contribute via a multiplicity of beneficial roles to the maintenance of good health, particularly by reducing the incidence of cardiovascular diseases. ${ }^{39,40}$

\section{Novel monounsaturated fatty acids}

The synthesis of common monounsaturated fatty acids is catalyzed by a soluble plastidial desaturase enzyme, which normally introduces a double bond between carbons 9 and 10 of a C18 acyl-ACP. ${ }^{11}$ However, some plants that are able to synthesize unusual monounsaturated fatty acids present an additional desaturase enzyme, which is closely related to the $\Delta^{9}$ desaturase, but introduces a double bond in positions other than the ninth carbon from the carboxyl group. ${ }^{10}$

Umbelliferae species, such as carrot and coriander, are known to contain oils rich in petroselenic acid $\left(\Delta^{6}\right.$ 18:1) (Figure $\left.2 C\right)$. This unusual monounsaturated fatty acid is the result of the activity of a plastidial $\Delta^{4}$ desaturase that introduces a double bond between carbons 4 and 5 of a C16 acyl-ACP, converting palmitoyl-ACP to $\Delta^{4}$ hexadecanoyl-ACP which is then elongated to petroselinoyl-ACP and cleaved from ACP to produce the free fatty acid. $^{41}$

Another curious example of this unusual desaturation process is observed in Meadowfoam (Limnanthes alba) oil. This specie accumulates oil with approximately $65 \%$ of $20: 1$ acid in its fatty acid composition. The 20:1 acid possesses a double bond at C5 carbon. ${ }^{42}$

\section{Hydroxy, epoxy and acetylenic fatty acids}

Fatty acids with additional functional groups in the acyl chain represent excellent feedstocks for industry and have been used to pro- duce innumerous bio-based products due to their physical and chemical properties. ${ }^{17}$

The synthesis of the fatty acids containing functional groups such as hydroxyl, epoxy and acetylenic functional groups is achieved by the action of a family of related enzymes. Structurally, these enzymes present similarities to extraplastidial membrane-bound $\Delta^{12}$-desaturases (FAD2), and only four amino acid substitutions are sufficient to convert an 18:1desaturase enzyme into an 18:1 hydroxylase enzyme. ${ }^{43}$

The synthesis of functional groups possessing fatty acids is thought to take place in the ER and uses as a substrate fatty acids esterified to the major membrane lipid phosphatidylcholine (PC). ${ }^{11}$

Castor oil, for example, is rich in hydroxylated fatty acid ( $\mathrm{OH})$ (Figure 2D), whereas some plants have epoxidated (Figure 2E) or methylated acyl chains in their TAGs. ${ }^{10}$ Examples of plants that accumulate these kinds of fatty acids include Vernonia galamensis, Euphorbia lagascae and Stokesia laevis that accumulate $60-80 \%$ of an epoxy fatty acid known as vernolic acid (cis-12-epoxyoctadeca-cis-9-enoic acid). ${ }^{43,44}$

\section{Triacylglycerols biosynthesis in plants}

TAGs are present in all eukaryotes, including animals, plants, fungi and protists, and also in some prokaryotes such Actinomycetes ${ }^{45-47}$ Streptomyces species ${ }^{48}$ and mycobacteria species. ${ }^{49}$ Due to its importance as an inert storage component, the biosynthesis of TAGs is a common metabolism pathway that appears to be conserved from bacteria to humans. ${ }^{50}$

TAGs are quantitatively the most important seed storage reserve in many plant species including oil crops such as sunflower 
(Helianthus annuus), oilseed rape (Brassica napus), soybean (Glycine max), and maize (Zea mays). ${ }^{43,51}$ Besides their importance as energy source, TAGs also represent an important source for building blocks for membrane lipid biosynthesis. ${ }^{50}$ TAGs from plants are important sources for human nutrition, also providing precursors for chemical industry products. ${ }^{14,43}$ TAGs are becoming increasingly important raw materials for the production of paints, detergents, lubricants, biofuels and nylon precursors, and can also serve as renewable biofuels as an alternative to crude oil. ${ }^{9}$

TAGs accumulated during seed maturation are stored in the seed oil bodies until germination $^{51-53}$ and can either be used for energy production through $\beta$-oxidation or serve as substrates for acylation reactions, such as phospholipid biosynthesis, for example. ${ }^{50}$

\section{Pathways for TAG biosynthesis}

In seed plants, glycerolipids can be synthesized via two similar pathways that are known as prokaryotic and eukaryotic systems on the basis of their evolutionary origins. These two pathways occur in distinct subcellular compartments and are similar in the two-step enzymatic conversion of sn-glycerol-3-phosphate (G3P) to phosphatidic acid (PA), but differ in the subsequent conversion of PA into structural, storage and signaling lipids. ${ }^{54}$ Although the two enzymatic pathways are similar, the enzymes that catalyze the acylations are unique to their respective system. ${ }^{55}$ Plastidic enzymes are responsible for the prokaryotic system, while cytosolic enzymes are responsible for the eukaryotic system. In the prokaryotic pathway, fatty acids are directly transferred from ACP to G3P, while in the eukaryotic pathway fatty acids are cleaved from the ACP by an acyl-ACP-thioesterase to form free fatty acids that are exported to the cytoplasm, esterified to $\mathrm{CoA}$ and then join the acyl-CoA pool. The acyl groups are then used by the acyltransferases of the eukaryotic G3P pathway in the ER to produce membrane and storage lipids (TAGs). ${ }^{11}$ Moreover, these enzymes can present structural differences depending on their subcellular localizations, forming independent clusters in phylogenetic studies. $^{56}$

Production of storage lipids in plants involves de novo fatty acid synthesis in the stroma of plastids and subsequent incorporation of the fatty acid into glycerol backbone leading to TAG in the ER. ${ }^{9}$

Two metabolic pathways for the production of TAGs have been clarified: an acyl CoAdependent pathway (Figure 1B) and an acylCoA-independent pathway (Figure 3).

\section{Acyl CoA-dependent pathway}

In the acyl-CoA dependent pathway, com- monly known as the Kennedy pathway (Figure 1B), acyl-CoA is the substrate for successive acylation reactions of the glycerol backbone, with the terminal step being the acylation of $s n$-1,2- diacylglycerol (DAG) by DAG acyltransferases (DGATs) ${ }^{57}$ The glycerol backbone for TAG assembly is derived from G3P which is produced via the catalytic action of $s n$-glycerol3-phosphate dehydrogenase (G3PDH) from dihydroxyacetone phosphate (DHAP), derived from glycolysis. ${ }^{18}$

The Kennedy pathway starts with acylation of G3P to form lysophosphatidic acid (LPA) through the action of $s n$-glycerol-3-phosphate acyltransferase (G3PAT). The second acyl-CoA dependent acylation is catalyzed through the catalytic action of lysophosphatidic acid acyltransferase (LPAAT), leading to the formation of phosphatidic acid (PA). Phosphatidic acid phosphatase (PAP) catalyzes the release of phosphate from PA to produce DAG. ${ }^{9}$ The final acylation is driven by DGAT, using acyl CoA as an acyl donor, converting DAG to TAG (Figure 1B). ${ }^{14}$

\section{Glycerol-3-phosphate acyltransferase}

Membrane-bound Glycerol-3-phosphate acyltransferase (G3PAT) is a soluble enzyme that initiates the fatty acid incorporation process by transferring fatty acids from either acyl-ACPs or acyl-CoA molecules to the sn-1 position of G3P, forming LPA. ${ }^{10}$

In several plants, two G3PAT isoforms have been found in the plastidial and cytoplasmic cellular compartments. G3PATs can either be selective, preferentially using oleic acid as the acyl donor, or non-selective, using either oleic or the saturated palmitic acid at comparable rates. This differential substrate specificity for saturated versus unsaturated fatty acids has been implicated in the sensitivity of plants to chilling temperatures. ${ }^{58}$

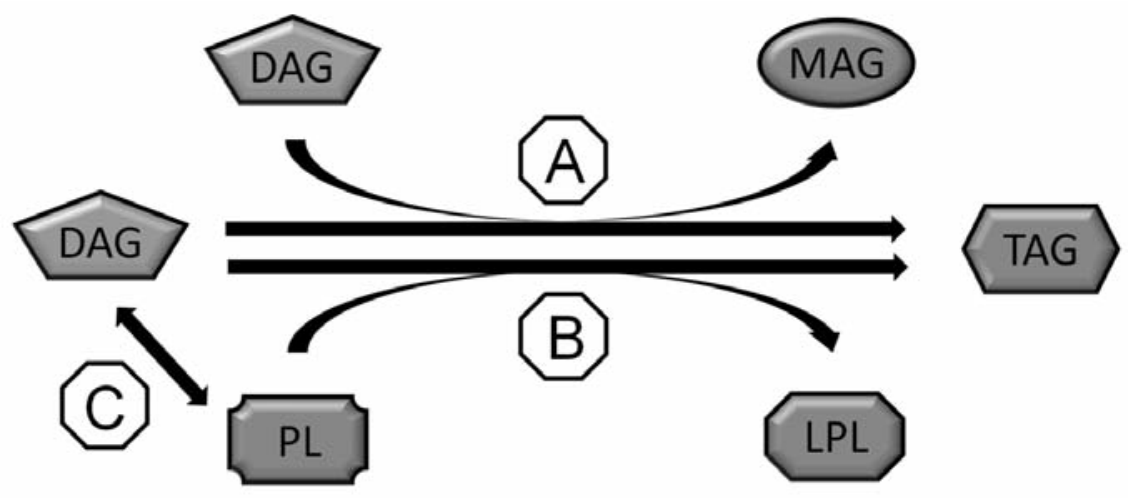

Figure 3. Acyl-CoA independent pathways for triacylglycerol (TAG) biosynthesis. Diacylglycerol transacylase (A) catalyzes the transfer of an acyl moiety between two diacylglicerol (DAG) molecules to form TAG and monoacylglycerol (MAG) as a co-product. Phospholipid: diacylglycrol acyltransferase (B) catalyzes the transfer of fatty acids from the sn-2 position of phospholipids (PL) to DAG to form TAG and lyso-phospholipids (LPL) as a co-product. Choline phosphotransferase (C) can contribute to TAG biosynthesis through a reversible conversion of PL into DAG. Then, the resulting DAG can be converted into TAG by the DGAT or PDAT enzymes.

\section{Lysophosphatidic acid acyltransferase}

Lysophosphatidic acid acyltransferase (LPAAT) catalyzes the transfer of the acyling to the formation of PA. There are two sequence-diverged gene subfamilies encoding LPAAT. ${ }^{10}$

LPAAT activity is associated, in plants, with multiple membrane systems, including chloroplasts, ER and the outer membrane of mitochondria, which suggests their presence on several different isoforms.$^{59,60}$ In plants, this enzyme shows a preference for unsaturated acyl chains,${ }^{10}$ but it is also able to discriminate acyl groups having longer or shorter fatty acid chain lengths. In developing seeds of certain plants, special LPAATs can incorporate unusual acyl groups, such as in Castor bean, which accumulates oils esterified with unusual fatty acids. $^{61}$

\section{Phosphatidic acid phosphatase}

The cytoplasmic enzyme Phosphatidic acid phosphatase (PAP) dephosphorylates PA to yield DAG. ${ }^{62}$ DAG produced from the hydrolysis of PA is not only a direct precursor of TAG, but also a substrate for the synthesis of membrane spholipids. ${ }^{63}$ In plants, two distinct PAP involved in glycerolipid synthesis appear ed fractions (microsomes). The distribution of these forms seems to be affected by the cellular metabolic status. ${ }^{64}$

PAP is also involved in general phospholipid degradation and turnover. It was demonstrated that PA accumulates in plants in a transient manner in response to various forms of stress. dephosphorylation activity of PAP results in the attenuation of the signaling function of PA through its conversion to DAG, which can 
be important in the stress responses, especially in the remodeling of membrane lipid composition. ${ }^{64,65}$

\section{Diacylglycerol acyltransferase}

Kennedy pathway's final enzyme, diacylglycerol acyltransferase (DGAT), catalyzes the third acyl-CoA-dependent acylation reaction that leads to the production of TAG from DAG. DGAT is an integral ER protein and has also been shown to be present in oil bodies and plastids. ${ }^{66}$ Although DGAT1 and DGAT2 are the main types of DGAT enzymes, other different, structurally unrelated enzymes with DGAT activity have been described in plants. ${ }^{67,68} \mathrm{~A}$ soluble DGAT from peanuts, ${ }^{69}$ a wax ester synthase/acyl-coenzyme A: Diacylglycerol acyltransferase (WSD1) from Arabidospis ${ }^{43}$ and a distinct DGAT (DAcT) from Euonymus alatus. ${ }^{70}$ The substrate selectivity of DGAT depends on several factors, such as the acyl composition of the DAG pool, acyl-CoA concentration and temperature. ${ }^{9}$

DGAT1 was initially cloned from mouse based on its homology with mammalian acylCoA: cholesterol acyltransferase genes. ${ }^{71}$ Several homologs of DGAT1 have been cloned and characterized in animals and plants, and their functions have been verified by both overexpression and deletion approaches. ${ }^{72-75}$

A second family of DGAT genes (DGAT2), which have no sequence similarity with DGAT1, were first identified in the oleaginous fungus Morteriella ramanniana. ${ }^{76,77}$ Several DGAT2 genes have been cloned and characterized from animals, fungi and plants. ${ }^{77-79}$ In animals, DGAT2 has different physiological functions in vivo and presents a different temporal-spatial expression profile compared to DGAT1.$^{80-82}$ In yeast, Sacharomyces cerevisae, the DGAT2 enzyme is dominant in the stationary growth phase when the yeast is storing significant amounts of TAG..$^{83,84}$ In addition, some experiments suggest a more important role of DGAT2 expression in the accumulation of conjugated and hydroxy fatty acid in seed oils. ${ }^{43,85-87}$

The roles of DGAT1 and DGAT2 in the oil production are apparently species-dependent. In plants, DGAT1 appears to be a major enzyme gene for seed oil accumulation, while DGAT2 appears to play significant roles in the selective accumulation of unusual fatty acids, such as epoxy and hydroxy fatty acid, into seed storage oils. ${ }^{43,86,88}$

A third enzyme is a soluble DGAT (DGAT3), which was only recently identified in peanut and other plant species. ${ }^{67,86}$ However, there is little information about this new DGAT isoform. An acyl-CoA-dependent acyltransferase, namely wax ester synthase/diacylglycerol acyltransferase (WS/DGAT), was identified and purified from the bacterium Acinetobacter sp. strain ADP1, which can utilize both fatty alcohols and DAG as acyl acceptors to synthesize wax esters and TAGs, respectively. ${ }^{88-90} \mathrm{~A}$ large number of genes with homology to this Acinetobacter gene ${ }^{79,91}$ were identified in Arabidopsis.

DGATs may be one of the rate-limiting steps in plant storage lipid accumulation, $9,88,92$ and thus appear to be crucial for mediating quantitative and qualitative aspects of seed oil synthesis in transgenic plants. ${ }^{67}$ In this scenario DGAT seems to be a potential target for the genetic modification of plant lipid biosynthesis in oilseeds for economic benefit. ${ }^{88}$ Studies in this field demonstrated that overexpression of DGAT1 resulted in increase, ${ }^{92}$ whereas suppression of DGAT activity resulted in a decrease in oil content in Arabidopsis seeds. ${ }^{75,93}$ In addition, DGAT activity seems to be also important for the correct channeling of unusual fatty acids into seed storage oils. ${ }^{67}$

\section{Acyl CoA-independent pathway}

As an alternative to the Kennedy pathway, nascent fatty acids may be first incorporated into membrane lipids at the plastid envelope and/or in the ER and afterwards accumulated as TAG molecules. ${ }^{14}$

Newly synthesized fatty acids can be incorporated directly into $\mathrm{PC}$ via an acyl editing mechanism, rather than through PA and DAG intermediates. ${ }^{94}$ Acyl chains from PC can be incorporated into TAG, either through conversion back to DAG or by the action of a phospholipid: diacylglycerol acyltransferase (Figure 3) (PDAT). ${ }^{14}$ This enzyme is a member of the lecithin: cholesterol acyltransferase gene family ${ }^{91}$ that catalyzes the formation of TAG by an acyl transfer from the $s n-2$ position of phospholipids to DAG (Figure 3), with phosphatidylethanolamine (PE) as the preferred acyl donor in both yeast and plants. ${ }^{95-97}$

PDAT activity has been reported in yeast microsomes and certain oilseeds. ${ }^{91,96,98}$ It was demonstrated that Arabidopsis PDAT is able to utilize different phospholipids as acyl donors and accept acyl groups of chain lengths ranging from $\mathrm{C} 10$ to $\mathrm{C} 22 .{ }^{43,97}$ In yeast, PDAT1 is a major contributor to TAG accumulation during the exponential growth phase. ${ }^{76,83}$

The activity of PDAT enzymes (specially in oilseed plants) may play a critical role in the removal of unusual fatty acids from membrane phospholipids and transfer it into TAG molecules. ${ }^{9}$ It was suggested that the balance of the expression of PDAT genes may represent an important mechanism either for the maintenance of membrane lipid homeostasis, contributing to membrane lipid turnover or for the removal of DAG, an effector molecule of the phosphatidylinositol signaling pathway that possesses a critical role for plant stress responses. $^{91}$

In another case of acyl-CoA-independent transacylation, it has been postulated that a DAG transacylase (DGTA) catalyzes the transfer of an acyl moiety between two DAG molecules to form TAG, and monoacylglycerol (MAG) as a co-product (Figure 3), ${ }^{85,99}$ and that the reverse reaction participates in the remodeling of TAGs. ${ }^{88,100}$ However, no gene encoding such as transacylase has been identified so far. ${ }^{85}$

There is growing evidence for the presence of an alternative acyl-independent pathway for TAG formation in plants, involving an enzyme that is normally related with membrane biosynthesis, named choline phosphotransferase (CPT) or amino-alcohol phosphotransferase (AAPT). ${ }^{10}$ CPT can contribute to TAG biosynthesis through a reversible conversion of PC to DAG (Figure 3). ${ }^{12}$ Afterwards, the resulting DAG can be converted into TAG by DGAT or PDAT enzymes. It has been hypothesized that the continuous reversible transfer of DAG into PC may control, in part, the PUFA content of the seed oil, ${ }^{101}$ making CPT the key enzyme regulating the route by which PUFAs are available for incorporation into TAG molecules. $^{22,101,102}$

In addition, a recent publication demonstrated that a class of phospholipase enzyme (phospholipase D) plays an important role in the conversion of PC into TAG. ${ }^{103}$ The attenuation by RNA interference of a soybean phospholipase D was responsible for higher levels of di18: 2 (dilinoleoyl)-PC and PE in seeds compared to the wild type lines. The increased polyunsaturation was at the expense of $\mathrm{PC}$ and PE species containing monounsaturated or saturated fatty acids. By contrast, a decrease in the unsaturation of the TAG fraction of the soybean seeds was observed, suggesting that phospholipase D suppression slows the conversion of PC into TAG. ${ }^{103}$

\section{Regulation of oil biosynthesis in plants}

The accumulation at high levels of compounds for nutrient storage is a characteristic event of seed development which is regulated by a common, at least in part, genetic program that takes place during the seed maturation phase. ${ }^{104-108}$ The regulation of oil synthesis occurs at multiple levels. ${ }^{109}$ Several of the enzymes involved in the synthesis, accumulation and degradation of neutral lipids have been identified and a great redundancy for most of the neutral lipid metabolic enzymes was observed. The proteins involved in neutral lipid metabolism are well conserved across species, exhibiting remarkable homology to each other. ${ }^{50}$

Synthesis and accumulation of storage com- 
pounds are regulated by numerous transcription factors (TFs) in an intricate network involving genetic programs, and hormonal and metabolic signals. ${ }^{110-112}$ Some of these TFs are known as master regulators based on their apparent capacity to regulate the action of other TFs. ${ }^{18}$ The most important master regulators of seed maturation and storage accumulation include the LEAFY COTYLEDON genes (LEC1 and LEC2), FUSCA3 (FUS3), ABSCISIC ACID INSENTIVE3 (ABI3) and WRINKLED1 (WRI1). ${ }^{18,111}$

Among the known transcriptional factors that regulate genes related to storage compounds, it was demonstrated that WRIl activity was responsible for the regulation of oil accumulation by promoting the carbon flux through the glycolysis pathway. ${ }^{113-116}$

WRIl encodes a transcription factor of the APETALA2-ethylene responsive element-binding protein (AP2-EREBP) family. ${ }^{114}$ This TF is responsible for specifying the regulatory action of other TFs, such as LEC2 and possibly LEC1, during the fatty acid biosynthetic network. ${ }^{110,117}$

Studies in Arabidopsis demonstrated that WRI1 up-regulates glycolytic gene expression required for the conversion of sucrose to TAG biosynthesis precursors. ${ }^{112,114}$ The corresponding wril mutant is deficient in oil biosynthesis and shows a reduction of $80 \%$ in seed oil content. $^{14,115}$ The carbohydrate metabolism regulation in wril appears to be affected because the activities of a number of key glycolytic enzymes were highly reduced in that mutant. ${ }^{18}$

Other transcription factors are also involved with the regulation of oil metabolism in developing oil seeds. ${ }^{14}$ It was shown that seed-specific overexpression of Arabidopsis LEC1 in developing seedlings of Arabidopsis, using an estradiol-inducible vector, up-regulates the transcription level of known enzyme-coding genes of fatty acid biosynthesis by about $60 \%{ }^{118}$ The same study showed that the amount of fatty acids was 4.7 times higher in transgenic than wild-type seedlings and that the function of LEC1 in fatty acid biosynthesis regulation was partially dependent on $\mathrm{ABI} 3$, FUS3 and WRI1. 18,118

FUS3 also seems to play an important role in oil accumulation in Arabidopsis. Transcriptomic analysis has revealed that the abundance of FUS3 transcript increases together with transcripts involved with fatty acid pathway. ${ }^{113}$ In addition, the inducible expression of Arabidopsis FUS3 resulted in rapid induction of gene expression associated with FA biosynthesis in seedlings. The same results were also observed in Arabidopsis protoplasts transiently expressing FUS3. ${ }^{18}$

Soybean DNA binding proteins with one zinc-finger motif, or Dof-type TFs, have also been shown to have effects on oil accumulation. Overexpression of GmDof4 and GmDof11 leads to an increased expression level of the genes encoding the $\beta$-subunit of acetyl $\mathrm{CoA}$ carboxylase (ACCase) and long-chain acyl-CoA synthetase, respectively, both encoding enzymes involved with fatty acid biosynthesis. In addition, the transgenic lines with the highest levels of GmDof4 or GmDof11 expression also present a lipid content increase ranging from 11 to $24 \%$. $^{18,119}$

However, besides the fatty acid biosynthesis regulation promoted by TFs, additional levels of control certainly involve allosteric enzyme regulation, for example, at the level of ACCase $^{15,120}$ or plastid pyruvate kinase, ${ }^{14,121,122}$ important precursors of TAG biosynthesis.

\section{Oil bodies}

Several organisms store lipids in subcellular particles as food reserves, which will be used through a period of active metabolism. These lipid particles can be found in seeds, pollens, flowers, roots, stems of flowering plants, spores and vegetative organs of non-flowering plants and algae. These structures are also represented in some animal cells, besides fungi and Euglena. However, seed subcellular storage lipid particles, have been studied more extensively. ${ }^{123}$

Seeds of most plant species store TAGs as food reserves for germination and post-germi-
A)

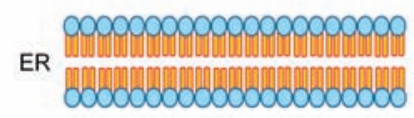

C)

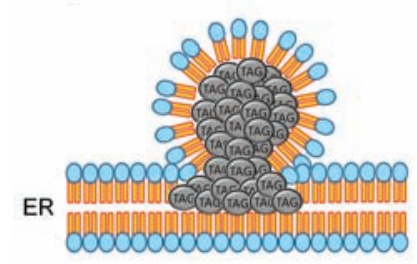

native growth. ${ }^{123}$ The intracellular storage of neutral lipids occurs in specialized compartments called lipid particles, lipid droplets or oil bodies. ${ }^{50}$

Oil bodies are relatively simple spherical organelles with approximately $1 \mu \mathrm{m}$ in diameter that arise from the ER, the site of TAG synthesis, and are surrounded by a phospholipid monolayer membrane embedded with proteins called oleosins..$^{51,124,125}$ Oleosins in the seeds of diverse species are small proteins of about 15 to 26 Kilodaltons ${ }^{123,126}$ that are usually present as two or more highly conserved isoforms. ${ }^{51,127}$

According to the best accepted oil body biogenesis model, proteins involved in neutral lipid metabolism accumulate in certain regions of the ER. Enzymes involved in TAGs formation are found among these polypeptides. ${ }^{50}$ TAGs are synthesized in the ER and are sequestered, due to their hydrophobicity, between the two layers of the ER membrane. ${ }^{123}$ Because newly formed neutral lipids are unable to integrate into bilayer membranes they cluster and accumulate in the hydrophobic region between the two leaflets of the ER membrane (Figure 4A and B). During ongoing TAG synthesis the droplet grows and forms a bud (Figure 4C). After reaching a certain size, the budding particle, which has a TAG matrix surrounded by a layer of phospholipids and oleosins, is released into the cytosol as a mature oil body (Figure 4D)..$^{50,123}$
B)

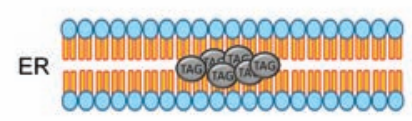

D)

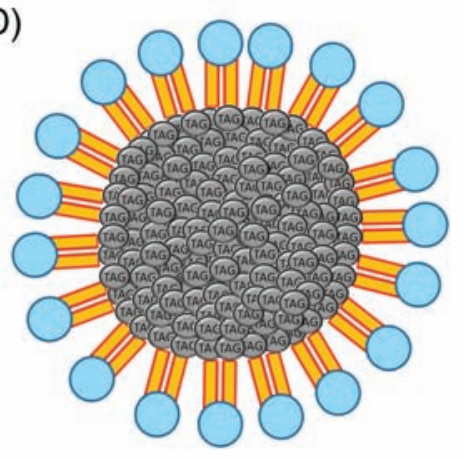

Figure 4. Oil body biosynthesis. (A-B) Accumulation of triacylglycerols (TAGs) occurs between the two layers of endoplasmic reticulum membrane (ER). (C) During ongoing synthesis of TAGs the droplet grows and forms a bud. (D) After reaching a certain size, the budding particle is released into the cytosol as a mature oil body. Adapted from Athenstaedt and Daum. ${ }^{50}$ 


\section{Lipid biosynthesis in algae}

Eukaryotic algae represent a very diverse organism group that is considered a key component for the most diverse ecosystems. They account for over half of the primary production at the base of food chains. ${ }^{128}$

Their variable morphology and habitats mean that eukaryotic algae contain a diverse composition of acyl lipids and unusual fatty acids that are not found in other phyla. ${ }^{128}$ It is widely accepted that the excellent capability of algae to adapt to very different environmental conditions results from the fact that they can synthesize a number of unusual compounds, responsible for their unusual pattern of cellular lipids, as well as their ability to modify efficiently the lipid metabolism in response to environmental conditional changes. ${ }^{129,130}$ Indeed, some authors suggested that the capacity of some algae to store VLC-PUFAs in TAG could be a reserve, which would allow the organisms to adapt to any further rapid changes in their environment, such as high light intensity, UV radiation and low temperature. ${ }^{131}$

Based on their lipid diversity, several thousands of algae and cyanobacterial species have been screened for high lipid content, resulting in the isolation and characterization of several hundred oleaginous species. These species can be found among diverse taxonomic groups, and the total lipid content may vary noticeably among individual species or strains, within and between taxonomic groups. ${ }^{132}$

\section{Comparison of lipid metabolism in algae and higher plants}

Lipid metabolism, specially the pathways to fatty acids and TAG biosynthesis, is less understood in algae than in higher plants. It is generally accepted that the basic pathways of fatty acid and TAG biosynthesis in algae are directly analogous to those demonstrated in higher plants based on the sequence homology and some shared biochemical characteristics of a number of genes and/or enzymes involved in lipid metabolism. ${ }^{132}$

However, there is some evidence of differences in algae lipid metabolism. Unlike higher plants, where individual classes of lipids may be synthesized and localized in a specific cell, tissue or organs (seeds or fruits), the complete pathway from carbon dioxide fixation to TAG synthesis and sequestration takes place within a single algal cell. ${ }^{132}$ After being synthesized, the accumulation of algal TAGs occurs in densely packed lipid bodies located in the cytoplasm of the algal cell, although the lipid body formation and accumulation might also occur in the inter-thylakoid space of the chloroplast in some green algae species, such as
Dunaliella bardawil. ${ }^{133}$ Higher plants cannot synthesize significant amounts of VLC-PUFAs above $\mathrm{C} 18$, whereas many algae, especially marine species, possess the ability to synthesize and accumulate large quantities of VLCPUFAs, such as EPA, DHA and ARA. ${ }^{134}$ In addition, it is hypothesized that in algae, the TAG biosynthesis pathway may play a more active role in the stress response, in addition to functioning as carbon and energy storage under environmental stress conditions. ${ }^{132}$

\section{Fatty acid biosynthesis in algae}

Algae synthesize fatty acids as building blocks for the formation of various types of lipids. Similar to higher plants, the most commonly synthesized fatty acids have chain lengths ranging from $\mathrm{C} 16$ to $\mathrm{C} 18$ (Table 1). ${ }^{136}$ In general, saturated and mono-unsaturated fatty acids are predominant in most algae examined. Indeed, the major saturated fatty acid is palmitic acid, while oleic acid is much less abundant than in higher plants. ${ }^{132}$

However, some algae and cyanobacteria are able to synthesize, as predominant FA species, medium-chain fatty acids (C10, $\mathrm{C} 12$ and $\mathrm{C} 14)$, whereas others are able to produce VLC-FA (>C20). For instance, $27-50 \%$ of all fatty acids found in the filamentous cyanobacterium Trichodesmium erythraeum are composed by a C10 fatty acid and up to nearly $70 \%$ of all fatty acids in the golden alga Prymnesium parvum are composed of $\mathrm{C} 14$ fatty acid. ${ }^{137}$ The distribution of individual fatty acids is quite distinct and tightly regulated. This control relates primarily to the function of acyl lipids in the membrane composition. ${ }^{128}$

Another distinguishing feature of some algae is the large amounts of VLC- PUFAs $(>18 \mathrm{C})$ as their major fatty acid components (Figure 5, Table 1), which resulted from several desaturation/elongation steps, promoted by

Palmitic acid De novo synthesis $(16: 0)$
$(18: 0)$

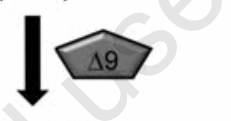

Oleic acid

$(18: 1)$

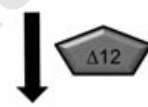

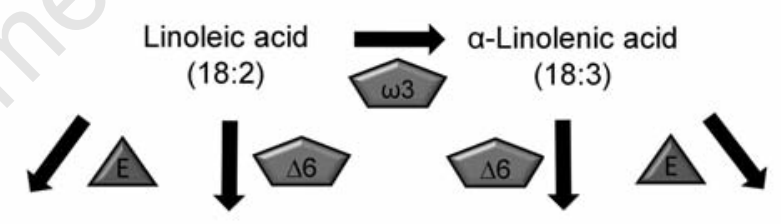

Eicosadienoic acid $(20: 2)$

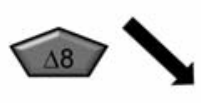

$\mathrm{\gamma}$-Linolenic acid

$(18: 3)$

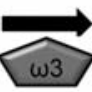

Stearidonic acid Eicosatrienoic acid

$(20: 3)$

Dihomo- $\gamma$-Linolenic acid Eicosatetraenoic acid (20:3) $(20: 4)$
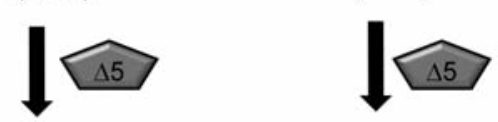

Arachidonic acid $(20: 4)$

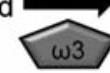

Eicosapentaenoic acid $(20: 5)$

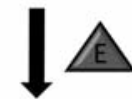

Docosahexaenoic acid $(22: 6)$

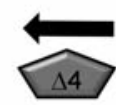

Docosapentaenoic acid $(22: 5)$

Figure 5. Very-long-chain polyunsaturated fatty acid (VLC-PUFA) biosynthesis in eukaryotic algae. Pentagons and triangles represent desaturases and elongases enzymes involved in algal VLC-PUFA biosynthesis, respectively. Adapted from Harwood and Guschina. ${ }^{128}$ 
large classes of desaturase and/or elongase enzymes (Figure 5). This is particularly true for marine species. Examples include the green alga Parietochloris incise ${ }^{131}$ the diatom Phaeodactylum tricornutum and the dinoflagellate Crypthecodinium cohnii ${ }^{138}$ where the VLC-PUFAs ARA, EPA or DHA constituted the major fatty acid species representing about $33.6-42.5 \%$, approximately $30 \%$ and $30-50 \%$ of the total fatty acid composition of these three species, respectively. In this scenario, the presence of $20 \%$ or more of three kinds of fatty acids in algae is attracting much economical interest because of the role of algae at the beginning of food chains and the perceived need for VLC-PUFAs in healthy diets. ${ }^{128}$

In addition, and alternatively to PUFAs, certain algae species may contain other unusual lipids in their oil composition, including chlorosulpholipids $^{139}$ and halogenated fatty acids. $^{140}$

\section{Triacylglycerols accumulation in algae}

TAG biosynthesis in algae may occur via the direct glycerol pathway (Kennedy pathway). ${ }^{141}$ The fatty acids that are produced in the chloroplast are sequentially transferred from CoA to positions 1 and 2 of G3P, by GPAT and LPAAT enzymes, respectively, forming $\mathrm{PA}{ }^{136} \mathrm{PA}$ is then dephosphorylated by a specific action of PAP enzyme releasing DAG. In the final step of TAG synthesis, a third fatty acid is transferred to the vacant position 3 of DAG, by the action of DGAT. As in plants, the acyltransferases involved in the TAG synthesis process seem to exhibit preferences for specific acyl-CoA, and thus may play an important role in determining the final acyl composition of TAG molecules. $^{132}$

Beyond the Kennedy pathway, the existence was suggested of an acyl-CoA-independent synthesis of TAG in algae, through PDAT and CPT activities, similar to that observed in plant and yeast. This pathway could play an important role in the regulation of the membrane lipid composition in response to various environmental and growth conditions, since under various stress conditions, algae usually undergo rapid degradation of the photosynthetic membrane with concomitant occurrence and accumulation of cytosolic TAG-enriched lipid bodies. ${ }^{132}$

Despite the occurrence and the levels of algal TAG production appear to be species/strain-specific, oleaginous algae produce only small quantities of TAG under optimal growth or favorable environmental conditions. ${ }^{142}$ Under optimal conditions of growth, algae are able to synthesize fatty acids mainly for esterification into glycerol-based membrane lipids, which represent about 5-20\% of their dry cell weight (DCW). The membrane

Table 1. Major fatty acid and polyunsaturated fatty acid (PUFA) in algae.

\begin{tabular}{|c|c|c|}
\hline Algal Class & Major Fatty Acid & Major PUFAs \\
\hline Bacillariophyceae & $\mathrm{C} 16: 0$ and $\mathrm{C} 16: 1$ & C20:5 and C22:6 \\
\hline Chlorophyceae & $\mathrm{C} 16: 0$ and $\mathrm{C} 18: 1$ & $\mathrm{C} 18: 2$ and $\mathrm{C} 18: 3$ \\
\hline Euglenophyceae & $\mathrm{C} 16: 0$ and $\mathrm{C} 18: 1$ & $\mathrm{C} 18: 2$ and $\mathrm{Cl} 8: 3$ \\
\hline Chrysophyceae & $\mathrm{C} 16: 0$ and $\mathrm{C} 16: 1$ and $\mathrm{C} 18: 1$ & C20:5, C22:5 and C22:6 \\
\hline Chryotophyceae & $\mathrm{C} 16: 0$ and $\mathrm{C} 20: 1$ & $\mathrm{C} 18: 3, \mathrm{C} 18: 4$ and $\mathrm{C} 20: 5$ \\
\hline Eustigmatophyceae & $\mathrm{C} 16: 0$ and $\mathrm{C} 18: 1$ & C20:3 and C20:4 \\
\hline Prasinophyceae & $\mathrm{C} 16: 0$ and $\mathrm{C} 18: 1$ & $\mathrm{C} 18: 3$ and $\mathrm{C} 20: 5$ \\
\hline Dinophyceae & $\mathrm{C} 16: 0$ & C18:5 and C22:6 \\
\hline Prymnesiophyceae & $\mathrm{C} 16: 0$ and $\mathrm{C} 16: 1$ and $\mathrm{C} 18: 1$ & $\mathrm{C} 18: 2, \mathrm{C} 18: 3$ and $\mathrm{C} 22: 6$ \\
\hline Rhodophyceae & C16:0 & C18:2 and C20:5 \\
\hline Xanthophyceae & C14:0, C16:0 and C16:1 & C 16:3 and C20:5 \\
\hline Cianobacteria & $\mathrm{C} 16: 0$ and $\mathrm{Cl}: 1$ and $\mathrm{C} 18: 1$ & $\mathrm{C} 16: 0, \mathrm{C} 18: 2$ and $\mathrm{C} 18: 3$ \\
\hline
\end{tabular}

lipid fatty acid composition includes mediumchain (C10-C14), long-chain (C16-18) and very-long-chain $(>\mathrm{C} 20)$ fatty acid derivatives. ${ }^{143}$

In contrast, under unfavorable environmental or stress conditions, many algae species promote a shift in lipid metabolism from membrane lipid synthesis to the synthesis and storage of neutral lipids, especially in the form of TAGs. The de novo biosynthesis and conversion of certain membrane polar lipids into TAGs may contribute to the overall increase in TAG content. As a result, TAGs may account for as much as $80 \%$ of the total lipid content in the cell. As an example of the shift in the lipid metabolism, many microalgae have the ability to produce substantial amounts (20-50\% DCW) of TAGs as lipid storage under photo-oxidative stress or other adverse environmental conditions. ${ }^{144}$

The major chemical stimuli to TAG accumulation in algae are nutrient starvation, salinity and growth-medium pH. On the other hand, the major physical stimuli are temperature and light intensity. In addition to chemical and physical factors, growth phase and/or aging of the culture also affects the content and fatty acid composition of TAG molecules. ${ }^{132}$

A significant variation in the algal lipid content and fatty acid profile in response to different growth conditions was observed. ${ }^{145-147}$ In Pavlova lutheri, a marine Pavlovophyceae, it was reported that the proportions of PUFAs, especially EPA, were significantly higher under low light. By contrast, content of saturated fatty acids and DHA were significantly higher under strong light. ${ }^{148}$ In addition, these authors also demonstrated that the growth and lipid composition presented a higher sensibility to variations in light intensity than in carbon source.

Another example of light effect in lipid and fatty acid composition includes Chlorella zofingiensis, a green algae that can grow well photoautotrophically as well as heterotrophically. It presented a $900 \%$ increase in lipid yield in heterotrophic cells compared with photoautotrophic cell culture. Moreover, about $80 \%$ of total lipid content was represented by neutral lipids in heterotrophic cells, with $88.7 \%$ being TAGs. On the other hand, photoautotrophic cells accumulated mainly membrane lipids (glycolipids and phospholipids). ${ }^{149}$

Guihéneuf and co-workers ${ }^{150}$ investigated the effect of UV radiation (UV-R) on the lipid composition of two marine microalgae, Pavlova lutheri and Odontella aurita. The results indicated that the exposure to UV-R treatment led to a decrease in the proportions of PUFAs especially into structural lipids (glycolipids and phospholipids) in $P$. lutheri, whereas in 0 . aurita, exposure to UV-R did not change the fatty acid composition and lipid fractions of the cells, suggesting that this species is more resistant and seems to be able to partially acclimate to UV-R. ${ }^{150}$

\section{Aplications of Triacylglycerols produced by plants and algae}

\section{Biodiesel}

Biodiesel is a clean-burning fuel derived from vegetable oils or animal fats, which has been used as alternative to diesel fuel. ${ }^{151}$ TAGs are the main components of the vegetable oils and animal fats that are used for biodiesel production. ${ }^{152}$

TAGs are the most similar chemical to fossil oil and, therefore, is the best potential replacement for the chemical industry. In fact, fossil oil is derived from ancient lipid-rich organic material, such as spores and planktonic algae that were sedimented and transformed under 
high pressure and temperature over millions of years. ${ }^{153}$ TAGs consist of several different fatty acids which present different physical and chemical properties. Therefore, the composition of these fatty acids will be the most important parameters influencing the corresponding properties of the biodiesel derived from TAGs. ${ }^{152}$

The advantages of the use of biodiesel include its higher oxygenated state compared to the conventional diesel, which leads to lower carbon monoxide (CO) production and reduced emission of particulate matter, and the few or lack of sulfur or aromatic compounds in its composition. These compounds are present in the conventional diesel and contribute to sulfur oxide and sulfuric acid formation, while aromatic compounds also increase particulate emissions and are considered carcinogens. ${ }^{14}$ Furthermore, the use of biodiesel confers additional advantages, including a higher flashpoint (allows safer handling and storage), faster biodegradation (particularly advantageous in environmentally sensitive areas where fuel leakage poses great hazards) and greater lubricity. ${ }^{14}$ However, the biggest environmental advantage of using biodiesel is that it is a renewable energy source, since the reduced hydrocarbon chains of biodiesel are derived from solar energy: plants and algae capture light energy during photosynthesis, converting carbon dioxide and water to the sugars, from which TAGs are derived. ${ }^{154}$

As a result of their rapid growth and substantial production of TAGs, algae could be employed as cell factories to produce oils and other lipids for biofuels and other biomaterials, such as biodiesel, methane, hydrogen, ethanol, among others. ${ }^{132}$ In this context, biofuel production using microalgae offers several advantages in relation to oilseed crops: a) the high growth rate and the high photosynthetic conversion efficiency of microalgae makes it possible to satisfy the demand for biofuels using limited land resources; b) the cultivation of microalgae consumes less water than land crops; c) microalgae thrive in saline/brackish water/coastal seawater for which there are few competing demands; d) microalgae are able to synthesize and to accumulate large quantities of neutral lipids, mainly TAGs; e) microalgae can utilize nutrients such as nitrogen and phosphorus from a variety of wastewater sources, providing additional benefits through wastewater bio-remediation; and f) are capable of tolerating high $\mathrm{CO}_{2}$ content in gas streams allowing high-efficiency of $\mathrm{CO}_{2}$ mitigation ${ }^{155,156}$ For these reasons, microalgae are capable of producing more oil per unit area of land, compared to terrestrial oilseed crops. ${ }^{157}$

On the other hand, one of the major drawbacks of microalgae for biofuel production is the low biomass concentration in the microalgal culture due to the limit of light penetration, which in combination with the small size of algal cells makes the harvest of algal biomasses relatively costly. ${ }^{155}$

The main limitation for the replacement of conventional diesel by biodiesel is that biodiesel still represents a small percentage of total diesel consumption, despite the large increase in its use. Two main factors have contributed to the limited adoption of biodiesel: problems with the fuel characteristics of biodiesel, namely poor cold-temperature properties, higher rates of oxidation and increased emission of nitrogen oxides (NOx) relative to the conventional diesel and the interrelated factors of cost and supply limitations, since the total world plant oil production, for example, would only satisfy approximately $80 \%$ of USA diesel demand. ${ }^{14}$

\section{Genetic manipulation of fatty acid content}

Modification of oil composition could contribute to the production of nutritionally and industrially desirable oils in crop plants and algae. ${ }^{64}$ The increasing global demand for oils has intensified the research efforts to genetically modify the organism to boost the oil yield. ${ }^{69}$ However, this requires not only a precise manipulation of fatty acid but also TAG synthesis in such a way that a specific synthesized fatty acid will be effectively incorporated into each position of TAG molecule. ${ }^{61}$

The natural diversity observed in seed and algae TAGs indicates that there should be no barriers to produce exotic FAs in domesticated oilseed crops and algae, and also provides a deep and potentially useful gene pool for genetic manipulation. ${ }^{10}$

Several unusual fatty acids described in this review, due to their unique chemical properties, have important industrial applications. However, to make these economically attractive, conventional oilseed crops will have to be genetically engineered to produce oils with a single predominant unusual fatty acid in its oil composition. The existence of wild species, such as castor, which contains oil with $90 \%$ hydroxylated fatty acid, suggests that this is a feasible task. ${ }^{11}$ Thus, attractive targets for plant genetic engineering for altered TAG composition are the temperate oilseed crops, such as soybean, rapeseed, flax, and sunflower. ${ }^{10}$

Today, the generation of transgenic crop plants engineered to accumulate high levels of specific unusual fatty acids is a topic of enormous interest. However, the inability to specifically target unusual fatty acids to seed TAGs, and their excessive accumulation in membrane lipids, might disrupt seed membrane integrity and impair seed development or germination. To overcome this problem it is essential to prevent the accumulation of unusual fatty acids in seed membrane lipids through engineering projects aimed at generating viable, high-yielding transgenic plants by introducing genes involved in the exclusion of unusual fatty acids from membranes. ${ }^{11}$

Oilseeds provide an attractive platform for the production of high-value fatty acids that can replace non-sustainable petroleum special chemicals, such as diesel ${ }^{67}$ However, in the case of the production of fatty acids in transgenic plants, the conversion of plant oils into biodiesel and chemical feedstocks competes with their use as food. Therefore, it is extremely important to ensure that these new industrial productions do not affect the supply of food at a time when world food requirements are increasing rapidly. ${ }^{14,110}$

On the other hand, the production of PUFAs by marine and freshwater microalgae is the subject of intensive research and increasing commercial attention. ${ }^{128}$ In this scenario, metabolic engineering through genetic manipulation might represent a promising strategy for the overproduction of algal oils. ${ }^{129}$

The focus of attention is the isolation and use of novel fatty acid metabolic genes, especially elongases and desaturases (Figure 5) that are responsible for PUFAs synthesis, from different species of algae and their transfer to plants in order to modify crops to create useful new products through genetic engeneering tools. ${ }^{26}$ One example of this tendency is the metabolic engineering of omega-3 long-chain polyunsaturated fatty acids in plants using an acyl-CoA $\Delta 6$-desaturase from the marine microalgae Micromonas pusilla, which originated an artificial pathway that produced $26 \%$ EPA in Nicotiana benthamiana leaves. ${ }^{158}$ The authors also demonstrated that this enzyme appears to function as an acyl-CoA desaturase that has preference for $\omega 3$ substrates, both in Arabidopsis and in yeast transgenic lines.

In this context, the identification and functional characterization of enzymes involved in biosynthesis of long-chain PUFAs in algae has an important biotechnological significance for the production of VLC-PUFAs in transgenic oilseed crops. ${ }^{159,161}$

In addition, it is believed that the most costly downstream processing steps in fuel production using microalgal is that most microalgae will not grow to a density higher than a few grams of biomass per liter of water. While there are several possible low-cost solutions to concentrate the biomass, these methods are slow and the resulting biomass may still require further dewatering. One possible solution is to manipulate the biology of microalgal cells to allow the secretion of fuels or feedstocks directly into the growth medium, through the manipulation of lipid secretory pathway. 156 
In conclusion, the complete clarification of enzyme activities responsible for controlling the flux of fatty acids to TAG will make a decisive contribution to the correct transfer of unusual fatty acids into storage oils and can generate new tools for genetic oil manipulation. ${ }^{39,153}$ In this scenario, TFs regulatory action can ultimately affect several reactions in biochemical pathways that contribute to the production and accumulation of storage compounds. Thus, modification of the expression of genes encoding TFs represents another interesting strategy that can be adopted in order to increase the accumulation of desirable oils in target organisms. ${ }^{18}$

\section{References}

1. Dormann P, Benning C. Galactolipids rule in seed plants. Trends in Plant Science 2002; 7:112-8.

2. Bertrand M. Carotenoid biosynthesis in diatoms. Photosynth Res 2010;106:89-102.

3. Lemoine Y, Schoefs B. Secondary ketocarotenoid astaxanthin biosynthesis in algae: a multifunctional response to stress. Photosynth Res 2010;106:155-77.

4. Wang XM. Plant phospholipases. Annual Review of Plant Physiology and Plant Molecular Biology 2001;52:211-+.

5. Meijer HJG, Munnik T. Phospholipid-based signaling in plants. Annual Review of Plant Biology 2003;54:265-306.

6. Dyas L, Goad LJ. STERYL FATTY ACYL ESTERS IN PLANTS (VOL 34, PG 17, 1993). Phytochemistry 1993;34:1663-3.

7. Pata, MO, Hannun YA, Ng CKY. Plant sphingolipids: decoding the enigma of the Sphinx. New Phytologist 2010;185:611-30.

8. Dunn TM, Lynch DV, Michaelson LV, et al. A post-genomic approach to understanding sphingolipid metabolism in Arabidopsis thaliana. Annals of Botany 2004;93:483-97.

9. Lung SC, Weselake RJ. Diacylglycerol acyltransferase: a key mediator of plant triacylglycerol synthesis. Lipids 2006;41:1073-88.

10. Voelker T, Kinney AJ. Variations in the Biosynthesis of Seed-Storage Lipids. Annu Rev Plant Physiol Plant Mol Biol 2001;52:33561.

11. Millar AA, Smith MA, Kunst L. All fatty acids are not equal: discrimination in plant membrane lipids. Trends Plant Sci 2000;5:95-101.

12. Somerville C, Browse J. Plant Lipids: Metabolism, Mutants, and Membranes. Science 1991;252:80-7.

13. Stymne SS. Triacylglycerol biosynthesis. In: The Biochemistry of Plants: A Comprehensive Treatise. Academic Press, Orlando, USA, 1987, pp. 175-214.

14. Durrett TP, Benning C, Ohlrogge J. Plant triacylglycerols as feedstocks for the production of biofuels. Plant J 2008;54:593-607.

15. Nikolau BJ, Ohlrogge JB, Wurtele ES. Plant biotin-containing carboxylases. Arch Biochem Biophys 2003;414:211-22.

16. Tai H, Jaworski JG. 3-Ketoacyl-acyl carrier protein synthase III from spinach (Spinacia oleracea) is not similar to other condensing enzymes of fatty acid synthase. Plant Physiol 1993;103:1361-7.

17. Dyer JM, Stymne S, Green AG, et al. Highvalue oils from plants. Plant $\mathrm{J}$ 2008;54:640-55.

18. Weselake RJ, Taylor DC, Rahman MH, et al. Increasing the flow of carbon into seed oil. Biotechnol Adv 2009;27:866-78.

19. Pollard MR, Anderson L, Fan C, et al. A specific acyl-ACP thioesterase implicated in medium-chain fatty acid production in immature cotyledons of Umbellularia californica. Arch Biochem Biophys 1991;284:306-12.

20. Mayer KM, Shanklin J. Identification of amino acid residues involved in substrate specificity of plant acyl-ACP thioesterases using a bioinformatics-guided approach. BMC Plant Biol 2007;7:1.

21. Salas JJ, Ohlrogge JB. Characterization of substrate specificity of plant FatA and FatB acyl-ACP thioesterases. Arch Biochem Biophys 2002;403:25-34.

22. Somerville $\mathrm{C}$, et al. Lipids: chapter 10 In BB Buchanan, W Gruissem, RL Jones, eds, Biochemistry and Molecular Biology of Plants. American Society of Plant Physiologists 2000:456-527.

23. Somerville C, et al. Lipids. In: BB Buchanan, W Gruissem, RL Jones (eds.) Biochemistry and Molecular Biology of Plants. American Society of Plant Physiologists, 2000, chap 10, p. 456-527.

24. van Erp H, Bates PD, Burgal J, et al. Castor phospholipid:diacylglycerol acyltransferase facilitates efficient metabolism of hydroxy fatty acids in transgenic Arabidopsis. Plant Physiol 2010;61:1092-106.

25. Damude HG, Kinney AJ. Enhancing plant seed oils for human nutrition. Plant Physiol 2008;147:962-8.

26. Napier JA. The production of unusual fatty acids in transgenic plants. Annu Rev Plant Biol 2007;58:295-319.

27. Napier JA, Graham IA. Tailoring plant lipid composition: designer oilseeds come of age. Curr Opin Plant Biol 2010;13:330-7.

28. Voelker TA, Worrell AC, Anderson L, et al. Fatty acid biosynthesis redirected to medium chains in transgenic oilseed plants. Science 1992;257:72-4.

29. Voelker TA, Worrell AC, Anderson L, et al. Broad-range and binary-range acyl-acyl-carrier protein thioesterases suggest an alternative mechanism for medium-chain production in seeds. Plant Physiol 1997;114:669-77.

30. Dehesh K, Edwards P, Fillatti J, et al. KAS IV: a 3-ketoacyl-ACP synthase from Cuphea sp. is a medium chain specific condensing enzyme. Plant J 1998;15: 383-90.

31. Graham SA. Cuphea: a new plant source of medium-chain fatty acids. Crit Rev Food Sci Nutr 1989;28(2):139-73.

32. Harwood JL. Recent advances in the biosynthesis of plant fatty acids. Biochim Biophys
Acta 1996;1301:7-56.

33. Napier JA, Sayanova 0. The production of very-long-chain PUFA biosynthesis in transgenic plants: towards a sustainable source of fish oils. Proc Nutr Soc 2005;64:387-93.

34. Wallis JG, Watts JL, Browse J. Polyunsaturated fatty acid synthesis: what will they think of next? Trends Biochem Sci 2002;27:467.

35. Williams CM, Burdge G. Long-chain $n-3$ PUFA: plant v. marine sources. Proc Nutr Soc 2006;65:42-50.

36. Domergue F, Abbadi A, Heinz E. Relief for fish stocks: oceanic fatty acids in transgenic oilseeds. Trends Plant Sci 2005;10:112-6.

37. Girke T, Schmidt H, Zähringer U, et al. Identification of a novel delta 6-acyl-group desaturase by targeted gene disruption in Physcomitrella patens. Plant J 1998;15:39-48.

38. Sayanova 0, Haslam R, Qi B, Lazarus CM, et al. The alternative pathway C20 Delta8-desaturase from the non-photosynthetic organism Acanthamoeba castellanii is an atypical cytochrome b5-fusion desaturase. FEBS Lett 2006;580:1946-52.

39. Abbadi A, Domergue F, Bauer J, et al. Biosynthesis of very-long-chain polyunsaturated fatty acids in transgenic oilseeds: constraints on their accumulation. Plant Cell 2004;16:2734-48.

40. Demaison L, Moreau D. Dietary n-3 polyunsaturated fatty acids and coronary heart disease-related mortality: a possible mechanism of action. Cell Mol Life Sci 2002;59:463-77.

41. Cahoon EB, Ohlrogge JB. Metabolic Evidence for the Involvement of a [delta]4-PalmitoylAcyl Carrier Protein Desaturase in Petroselinic Acid Synthesis in Coriander Endosperm and Transgenic Tobacco Cells. Plant Physiol 1994;104:827-37.

42. Metzger J0, Bornscheuer U. Lipids as renewable resources: current state of chemical and biotechnological conversion and diversification. Appl Microbiol Biotechnol 2006;71:1322.

43. Li R, Yu K, Hildebrand DF. DGAT1, DGAT2 and PDAT expression in seeds and other tissues of epoxy and hydroxy fatty acid accumulating plants. Lipids 2010;45:145-57.

44. Bafor M, Smith MA, Jonsson L, et al. Biosynthesis of vernoleate (cis-12-epoxyoctadeca-cis-9-enoate) in microsomal preparations from developing endosperm of Eu-phorbia lagascae. Arch Biochem Biophys 1993; 303:145-51.

45. Alvarez HM, Kalscheuer R, Steinbuchel A. Accumulation and mobilization of storage lipids by Rhodococcus opacus PD630 and Rhodococcus ruber NCIMB 40126. Appl Microbiol Biotechnol 2000;54:218-23.

46. Alvarez HM, Souto MF, Viale A, et al. Biosynthesis of fatty acids and triacylglycerols by 2,6,10,14-tetramethyl pentadecanegrown cells of Nocardia globerula 432. FEMS Microbiol Lett 2001;200:195-200.

47. Alvarez HM, Steinbuchel A. Triacylglycerols in prokaryotic microorganisms. Appl Microbiol Biotechnol 2002;60:367-76. 
48. Olukoshi ER, Packter MN. Importance of stored triacylglycerols in Streptomyces: possible carbon source for antibiotics. Microbiology 1994;140 (:931-43.

49. Akao T, Kusaka T. Solubilization of diglyceride acyltransferase from the membrane of Mycobacterium smegmatis. J Biochem 1976; 80:723-8.

50. Athenstaedt K, Daum G. The life cycle of neutral lipids: synthesis, storage and degradation. Cell Mol Life Sci 2006;63:1355-69.

51. Graham IA. Seed storage oil mobilization. Annu Rev Plant Biol 2008;59:115-42.

52. Hsieh K, Huang AH. Endoplasmic reticulum, oleosins, and oils in seeds and tapetum cells. Plant Physiol 2004;136:3427-34.

53. Murphy DJ, Vance J. Mechanisms of lipidbody formation. Trends Biochem Sci 1999;24: 109-15.

54. Banas A, Dahlqvist A, Ståhl U, et al. The involvement of phospholipid:diacylglycerol acyltransferases in triacylglycerol production. Biochem Soc Trans 2000;28:703-5.

55. Kim HU, Li Y, Huang AH. Ubiquitous and endoplasmic reticulum-located lysophosphatidyl acyltransferase, LPAT2, is essential for female but not male gametophyte development in Arabidopsis. Plant Cell 2005;17: 1073-89.

56. Cagliari A, Pinheiro-Margis M, Loss G, et al. Identification and expression analysis of castor bean (Ricinus communis) genes encoding enzymes from the triacylglycerol biosynthesis pathway. Plant Science 2010;179:499509.

57. Kennedy EP. Biosynthesis of complex lipids. Fed Proc 1961;20:934-40.

58. Turnbull AP, Rafferty JB, Sedelnikova SE, et al. Analysis of the structure, substrate specificity, and mechanism of squash glycerol-3phosphate (1)-acyltransferase. Structure 2001;9:347-53.

59. Bourgis F, Kader JC, Barret P, et al. A plastidial lysophosphatidic acid acyltransferase from oilseed rape. Plant Physiol 1999;120:913-22.

60. Yu B, Wakao S, Fan J, et al. Loss of plastidic lysophosphatidic acid acyltransferase causes embryo-lethality in Arabidopsis. Plant Cell Physiol 2004;45:503-10.

61. Weier D, Luhs WJ, Dettendorfer J, et al. sn-1Acylglycerol-3-phosphate acyltransferase of Escherichia coli causes insertion of cis-11 eicosenoic acid into the sn-2 position of transgenic rapeseed oil. Molecular Breeding 1998;4:39-46.

62. Franca MG, Matos AR, Darcy-Lameta A, et al. Cloning and characterization of droughtstimulated phosphatidic acid phosphatase genes from Vigna unguiculata. Plant Physiol Biochem 2008;46:1093-100.

63. Nakamura Y, Tsuchiya M, Ohta H. Plastidic phosphatidic acid phosphatases identified in a distinct subfamily of lipid phosphate phosphatases with prokaryotic origin. J Biol Chem 2007;282:29013-21.

64. Kocsis MG, Weselake RJ. Phosphatidate phosphatases of mammals, yeast, and higher plants. Lipids 1996;31:785-802.
65. Pierrugues 0, Brutesco C, Oshiro J, et al. Lipid phosphate phosphatases in Arabi-dopsis. Regulation of the AtLPP1 gene in response to stress. J Biol Chem 2001;276: 20300-8.

66. Siloto RM, Truska M, Brownfield D, et al. Directed evolution of acyl-CoA:diacylglycerol acyltransferase: Development and characterization of Brassica napus DGAT1 mutagenized libraries. Plant Physiol Biochem 2009;47:456-61.

67. Cahoon EB, Shockey JM, Dietrich CR, et al. Engineering oilseeds for sustainable production of industrial and nutritional feedstocks: solving bottlenecks in fatty acid flux. Curr Opin Plant Biol 2007;10:236-44.

68. Turkish AR, Henneberry AL, Cromley D, et al. Identification of two novel human acyl-CoA wax alcohol acyltransferases: members of the diacylglycerol acyltransferase 2 (DGAT2) gene superfamily. J Biol Chem 2005;280: 14755-64.

69. Saha S, Enugutti B, Rajakumari S, et al. Cytosolic triacylglycerol biosynthetic pathway in oilseeds. Molecular cloning and expression of peanut cytosolic diacylglycerol acyltransferase. Plant Physiol 2006;141:1533-43.

70. Durrett TP, McClosky DD, Tumaney AW, et al. A distinct DGAT with sn-3 acetyltransferase activity that synthesizes unusual, reducedviscosity oils in Euonymus and transgenic seeds. Proc Natl Acad Sci USA 2010;107:94649.

71. Cases S, Smith SJ, Zheng YW, et al. Identification of a gene encoding an acyl CoA:diacylglycerol acyltransferase, a key enzyme in triacylglycerol synthesis. Proc Natl Acad Sci USA 1998;95:13018-23.

72. Bouvier-Nave P, Benveniste P, Oelkers P, et al. Expression in yeast and tobacco of plant cDNAs encoding acyl CoA:diacylglycerol acyltransferase. Eur J Biochem 2000;267:85-96.

73. Hobbs DH, Lu C, Hills MJ. Cloning of a cDNA encoding diacylglycerol acyltransferase from Arabidopsis thaliana and its functional expression. FEBS Lett 1999;452:145-9.

74. Katavic V, Reed DW, Taylor DC, et al. Alteration of seed fatty acid composition by an ethyl methanesulfonate-induced mutation in Arabidopsis thaliana affecting diacylglycerol acyltransferase activity. Plant Physiol 1995;108:399-409.

75. Zou J, Wei Y, Jako C, et al. The Arabidopsis thaliana TAG1 mutant has a mutation in a diacylglycerol acyltransferase gene. Plant J 1999;19:645-53.

76. Zhang M, Fan J, Taylor DC, et al. DGAT1 and PDAT1 acyltransferases have overlapping functions in Arabidopsis triacylglycerol biosynthesis and are essential for normal pollen and seed development. Plant Cell 2009;21:3885-901.

77. Lardizabal KD, Mai JT, Wagner NW, et al. DGAT2 is a new diacylglycerol acyltransferase gene family: purification, cloning, and expression in insect cells of two polypeptides from Mortierella ramanniana with diacylglycerol acyltransferase activity. J Biol Chem
2001:276:38862-9.

78. Cases S, Stone SJ, Zhou P, et al. Cloning of DGAT2, a second mammalian diacylglycerol acyltransferase, and related family members. J Biol Chem 2001;276:38870-6.

79. Beisson F, Koo AJ, Ruuska S, et al. Ara-bidopsis genes involved in acyl lipid metabolism. A 2003 census of the candidates, a study of the distribution of expressed sequence tags in organs, and a web-based database. Plant Physiol 2003;132:681-97.

80. Yu XX, Murray SF, Pandey SK, et al. Antisense oligonucleotide reduction of DGAT2 expression improves hepatic steatosis and hyperlipidemia in obese mice. Hepatology 2005;42:362-71.

81. Stone SJ, Myers HM, Watkins SM, et al. Lipopenia and skin barrier abnormalities in DGAT2-deficient mice. J Biol Chem 2004; 279:11767-76.

82. Orland MD, Anwar K, Cromley D, et al. Acyl coenzyme A dependent retinol esterification by acyl coenzyme A: diacylglycerol acyltransferase 1. Biochim Biophys Acta 2005;1737:7682.

83. Oelkers P, Cromley D, Padamsee M, et al. The DGAl gene determines a second triglyceride synthetic pathway in yeast. J Biol Chem 2002;277:8877-81.

84. Sandager L, Gustavsson MH, Stahl U, et al. Storage lipid synthesis is non-essential in yeast. J Biol Chem 2002;277:6478-82.

85. Kroon JT, Wei W, Simon WJ, et al. Identification and functional expression of a type 2 acyl-CoA:diacylglycerol acyltransferase (DGAT2) in developing castor bean seeds which has high homology to the major triglyceride biosynthetic enzyme of fungi and animals. Phytochemistry 2006;67:2541-9.

86. Shockey JM, Gidda SK, Chapital DC, et al. Tung tree DGAT1 and DGAT2 have nonredundant functions in triacylglycerol biosynthesis and are localized to different subdomains of the endoplasmic reticulum. Plant Cell 2006;18:2294-313.

87. Burgal J, Shockey J, Lu C, et al. Metabolic engineering of hydroxy fatty acid production in plants: RcDGAT2 drives dramatic increases in ricinoleate levels in seed oil. Plant Biotechnol J 2008;6:819-31.

88. Xu J, Francis T, Mietkiewska E, et al. Cloning and characterization of an acyl-CoA-dependent diacylglycerol acyltransferase 1 (DGAT1) gene from Tropaeolum majus, and a study of the functional motifs of the DGAT protein using site-directed mutagenesis to modify enzyme activity and oil content. Plant Biotechnol J 2008;6:799-818.

89. Kalscheuer R, Luftmann H, Steinbuchel A. Synthesis of novel lipids in Saccharomyces cerevisiae by heterologous expression of an unspecific bacterial acyltransferase. Appl Environ Microbiol 2004;70:7119-25.

90. Stoveken T, Kalscheuer R, Malkus U, et al. The wax ester synthase/acyl coenzyme A:diacylglycerol acyltransferase from Acineto-bacter sp. strain ADP1: characterization of a novel type of acyltransferase. J Bacteriol 
2005;187:1369-76.

91. Mhaske V, Beldjilali K, Ohlrogge J, et al. Isolation and characterization of an Arabidopsis thaliana knockout line for phospholipid: diacylglycerol transacylase gene (At5g13640). Plant Physiol Biochem 2005;43:413-7.

92. Jako C, Kumar A, Wei Y, et al. Seed-specific over-expression of an Arabidopsis cDNA encoding a diacylglycerol acyltransferase enhances seed oil content and seed weight. Plant Physiol 2001;126:861-74.

93. Routaboul JM, Benning C, Bechtold N, et al. The TAG1 locus of Arabidopsis encodes for a diacylglycerol acyltransferase. Plant Physiol Biochem 1999;37:831-40.

94. Bates PD, Ohlrogge JB, Pollard M. Incorporation of newly synthesized fatty acids into cytosolic glycerolipids in pea leaves occurs via acyl editing. J Biol Chem 2007;282:31206-16.

95. Ghosal A, Banas A, Stahl U, et al. Saccharomyces cerevisiae phospholipid:diacylglycerol acyl transferase (PDAT) devoid of its membrane anchor region is a soluble and active enzyme retaining its substrate specificities. Biochim Biophys Acta 2007;1771: 1457-63.

96. Dahlqvist A, Stahl U, Lenman M, et al. Phospholipid:diacylglycerol acyltransferase: an enzyme that catalyzes the acyl-CoA-independent formation of triacylglycerol in yeast and plants. Proc Natl Acad Sci USA 2000; 97:6487-92.

97. Stahl U, Carlsson AS, Lenman M, et al. Cloning and functional characterization of a phospholipid:diacylglycerol acyltransferase from Arabidopsis. Plant Physiol 2004; 135:1324-35.

98. Oelkers P, Tinkelenberg A, Erdeniz N, et al. A lecithin cholesterol acyltransferase-like gene mediates diacylglycerol esterification in yeast. J Biol Chem 2000275:15609-12.

99. Stobart K, Mancha M, Lenman M, et al. Triacylglycerols are synthesized and utilized by transacylation reactions in microsomal preparations of developing safflower (Carthamus tinctorius L.). Planta,1997;203: 58-66.

100. Lehner R, and Kuksis A. Biosynthesis of triacylglycerols. Prog Lipid Res 1996;35:169-201.

101. McMaster CR, Bell RM. CDP-choline:1,2-diacylglycerol cholinephosphotransferase. Biochim Biophys Acta 1997;1348:100-10.

102. Stobart AK, and Stymne S. The interconversion of diacylglycerol and phosphatidylcholine during triacylglycerol production in microsomal preparations of developing cotyledons of safflower (Carthamus tinctorius L.). Biochem J 1985;232:217-21.

103. Lee J, Welti R, Schapaugh WT, et al. Phospholipid and triacylglycerol profiles modified by PLD suppression in soybean seed. Plant Biotechnol J 2011;9:359-72.

104. Parcy F, Valon C, Kohara A, et al. The ABSCISIC ACID-INSENSITIVE3, FUSCA3, and LEAFY COTYLEDON1 loci act in concert to control multiple aspects of Arabidopsis seed development. Plant Cell 1997;9:1265-77.

105. Kagaya $Y$, Toyoshima R, Okuda R, et al. LEAFY COTYLEDON1 controls seed storage protein genes through its regulation of FUSCA3 and ABSCISIC ACID INSENSITIVE3. Plant Cell Physiol 2005;46:399-406.

106. Meinke DW. A Homoeotic Mutant of Arabidopsis thaliana with Leafy Cotyledons. Science 1992;258:1647-50.

107. Meinke DW, et al. Leafy Cotyledon Mutants of Arabidopsis. Plant Cell 1994;6:1049-1064.

108. Raz V, Bergervoet JH, Koornneef M. Sequential steps for developmental arrest in Arabidopsis seeds. Development 2001;128:243-52.

109. Hills MJ. Control of storage-product synthesis in seeds. Curr Opin Plant Biol 2004;7:3028.

110. Baud S, Lepiniec L. Physiological and developmental regulation of seed oil production. Prog Lipid Res, 2010;49:235-49.

111. Santos-Mendoza M, Dubreucq B, Baud S, et al. Deciphering gene regulatory networks that control seed development and maturation in Arabidopsis. Plant J 2008;54:608-20.

112. Gutierrez L, Van Wuytswinkel 0, Catelain M, et al. Combined networks regulating seed maturation. Trends Plant Sci 2007;12:294300.

113. Wang H, Guo J, Lambert Kn, et al. Deve-lopmental control of Arabidopsis seed oil biosynthesis. Planta 2007;226:773-83.

114. Cernac A, Benning C. WRINKLED1 encodes an AP2/EREB domain protein involved in the control of storage compound biosynthesis in Arabidopsis. Plant J 2004;40:575-85

115. Focks N, Benning C. wrinkled1: A novel, lowseed-oil mutant of Arabidopsis with a deficiency in the seed-specific regulation of carbohydrate metabolism. Plant Physiol 1998; 118:91-101.

116. Lara P, Onate-Sanchez L, Abraham Z, et al. Synergistic activation of seed storage protein gene expression in Arabidopsis by $\mathrm{ABI} 3$ and two bZIPs related to OPAQUE2. J Biol Chem 2003;278:21003-11.

117. Baud S, Mendoza MS, To A, et al. WRINKLED1 specifies the regulatory action of LEAFY COTYLEDON2 towards fatty acid metabolism during seed maturation in Arabidopsis. Plant J 2007;50:825-38.

118. Mu J, Tan H, Zheng Q, et al. LEAFY COTYLEDON1 is a key regulator of fatty acid biosynthesis in Arabidopsis. Plant Physiol 2008; 148:1042-54.

119. Wang HW, Zhang HW, Hao YJ, et al. The soybean Dof-type transcription factor genes, GmDof4 and GmDof11, enhance lipid content in the seeds of transgenic Arabidopsis plants. Plant J 2007;52:716-29.

120. Hunter SC, Ohlrogge JB. Regulation of spinach chloroplast acetyl-CoA carboxylase. Arch Biochem Biophys 1998;359:170-8.

121. Andre C, Froehlich TE, Moll MR, et al. A heteromeric plastidic pyruvate kinase complex involved in seed oil biosynthesis in Arabidopsis. Plant Cell 2007;19:2006-22.

122. Baud S, Wiulleme S, Dubreucq B, et al.
Function of plastidial pyruvate kinases in seeds of Arabidopsis thaliana. Plant $\mathrm{J}$ 2007;52:405-19.

123. Huang AH. Oleosins and oil bodies in seeds and other organs. Plant Physiol 1996;110: 1055-61.

124. Wallis JG, Browse J. Lipid biochemists salute the genome. Plant J 2010;61:1092-106.

125. Kim HU, Hsieh K, Ratnayake C, et al. A novel group of oleosins is present inside the pollen of Arabidopsis. J Biol Chem 2002;277:2267784.

126. Murphy DJ. Structure, function and biogenesis of storage lipid bodies and oleosins in plants. Prog Lipid Res 1993;32:247-80.

127. Tzen JT, Lai YK, Chan KL, et al. Oleosin isoforms of high and low molecular weights are present in the oil bodies of diverse seed species. Plant Physiol 1990;94:1282-9.

128. Harwood JL, Guschina IA. The versatility of algae and their lipid metabolism. Biochimie 2009;91:679-84.

129. Guschina IA, Harwood JL. Lipids and lipid metabolism in eukaryotic algae. Prog Lipid Res 2006;45:160-86.

130. Thompson GA. Lipids and membrane function in green algae. Biochim Biophys Acta 1996;1302:17-45.

131. Bigogno C, Khozin-Goldberg I, Boussiba S, et al. Lipid and fatty acid composition of the green oleaginous alga Parietochloris incisa, the richest plant source of arachidonic acid. Phytochemistry 2002;60:497-503.

132. Hu Q, Sommerfield M, Jarvis E, et al. Microalgal triacylglycerols as feedstocks for biofuel production: perspectives and advances. Plant J 2008;54:621-39.

133. Ben-Amotz A, Shaish A, Avron M. Mode of Action of the Massively Accumulated betaCarotene of Dunaliella bardawil in Protecting the Alga against Damage by Excess Irradiation. Plant Physiol 1989;91:1040-3.

134. Riekhof WR, Sears BB, Benning C. Annotation of genes involved in glycerolipid biosynthesis in Chlamydomonas reinhardtii: discovery of the betaine lipid synthase BTA1Cr. Eukaryot Cell 20054:242-52.

135. Cobelas MAL. Lipids in microalgae. I. Biochemistry. Grasas y Aceites 1989;40:118-145.

136. Ohlrogge J, Browse J. Lipid biosynthesis. Plant Cell 1995;7:957-70.

137.Parker, P.L., C. Van Baalen, L. Maurer, Fatty acids in eleven species of blue-green algae: geochemical significance. Science 1967;155:707-8

138. De Swaaf ME, de Ruk TC, Eggink G, Sijtsma L. Optimisation of docosahexaenoic acid production in batch cultivation by Crypthecodinium cohnii. J Biotechnol 1999;70:185-92.

139. Haines T. Sulpholipids and halosulpholipids. In: Biomembranes of Eukaryotic Microorganisms. JA Erwin (ed.) New York, 1973, p. 197-232.

140.Dembitsky VM, Srebnik M. Natural halogenated fatty acids: their analogues and derivatives. Prog Lipid Res 2002;41:315-67.

141. Ratledge C. An overview of microbial lipids. 
In: In Microbial

Lipids. Academic Press, New York, 1988, p. 3-11.

142. Hu Q. Environmental effects on cell composition. In: Handbook

of Microalgal Culture. Blackwell, Oxford, 2004, p. 83-93.

143. Pohl P, Wagner H. Control of fatty acid and lipid biosynthesis in Euglena gracilis by ammonia, light and DCMU. Z Naturforsch B 1972;27:53-61.

144.Tonon T. Long chain polyunsaturated fatty acid production and partitioning to triacylglycerols in four microalgae. Phytochemistry 2002;61:15-24.

145. Yongmanitchai W, Ward OP. Growth of and omega-3 fatty acid production by Phaeodactylum tricornutum under different culture conditions. Appl Environ Microbiol 1991; 57:419-25.

146. Carvalho AP, Pontes I, Gaspar H, et al. Metabolic relationships between macro- and micronutrients, and the eicosapentaenoic acid and docosahexaenoic acid contents of Pavlova lutheri. Enzyme and Microbial Technology 2006;38:358-66.

147. Petkov G, Garcia G. Which are fatty acids of the green alga Chlorella? Biochemical Systematics and Ecology 2007;35:281-5.

148. Guiheneuf F, Mimouni V, Ulmann L, et al. Combined effects of irradiance level and carbon source on fatty acid and lipid class composition in the microalga Pavlova lutheri commonly used in mariculture. Journal of Experimental Marine Biology and Ecology 2009;369:136-43.

149. Liu J, Huang J, Sun Z, et al. Differential lipid and fatty acid profiles of photoautotrophic and heterotrophic Chlorella zofingiensis: assessment of algal oils for biodiesel production. Bioresour Technol 2011;102:106-10.

150. Guiheneuf F, Fouqueray M, Mimouni V, et al. Effect of UV stress on the fatty acid and lipid class composition in two marine microalgae Pavlova lutheri (Pavlovophyceae) and Odontella aurita (Bacillariophyceae). Journal of Applied Phycology 2010;22:629-638.

151. Vasudevan PT, Briggs M. Biodiesel production--current state of the art and challenges. $\mathrm{J}$ Ind Microbiol Biotechnol 2008;35:421-30.

152. Lam MK, Lee KT, Mohamed AR. Homogeneous, heterogeneous and enzymatic catalysis for transesterification of high free fatty acid oil (waste cooking oil) to biodiesel: a review. Biotechnol Adv 2010;28:500-18.

153. Dyer JM, Mullen RT. Engineering plant oils as high-value industrial feedstocks for biorefining: the need for underpinning cell biology research. Physiol Plant 2008;132:11-22.

154. Hill J, Nelson E, Tilman D, et al. Environmental, economic, and energetic costs and benefits of biodiesel and ethanol biofuels. Proc Natl Acad Sci USA 2006;103:11206-10.

155. Li Y. Biofuels from microalgae. Biotechnol Prog 2008;24:815-20.
156. Radakovits, R., Jinkerson RE, Darzins A, et al., Genetic engineering of algae for enhanced biofuel production. Eukaryot Cell 2010; 9:486-501.

157. Huerlimann R, Nys R, Heimann K. Growth, lipid content, productivity, and fatty acid composition of tropical microalgae for scaleup production. Biotechnol Bioeng 2010;107: 245-57.

158. Petrie JR, Shrestha P, Mansour MP, et al. Metabolic engineering of omega-3 longchain polyunsaturated fatty acids in plants using an acyl-CoA Delta6-desaturase with omega3-preference from the marine microalga Micromonas pusilla. Metab Eng 2010;12:233-40.

159. Meyer A, Kirsch H, Domergue F, et al. Novel fatty acid elongases and their use for the reconstitution of docosahexaenoic acid biosynthesis. J Lipid Res 2004;45:1899-909.

160. Domergue F, Abbadi A, Zähringer U, et al. In vivo characterization of the first acyl-CoA Delta6-desaturase from a member of the plant kingdom, the microalga 0streococcus tauri. Biochem J 2005;389:483-90.

161. Drexler H, Spiekermann P, Meyer A, Domergue F, et al. Metabolic engineering of fatty acids for breeding of new oilseed crops: strategies, problems and first results. J Plant Physiol 2003;160:779-802. 